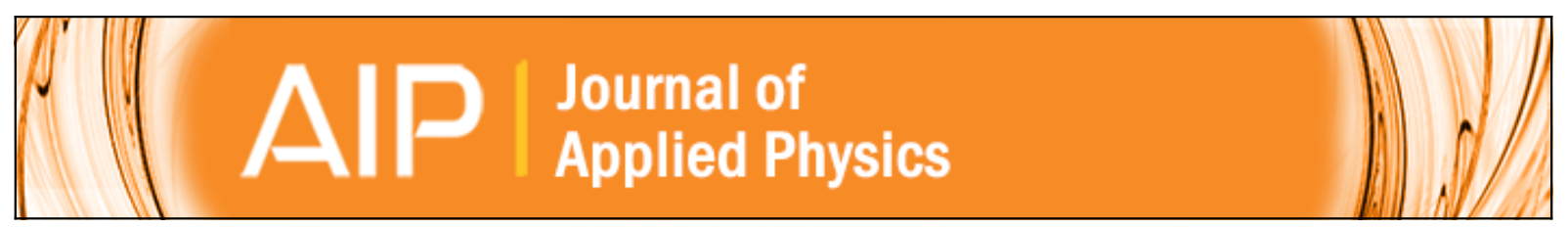

\title{
Magnetic, transport, and structural properties of SrRuO3 thin films
}

A. M. Majcher, K. Rode, J. M. D. Coey, and P. Stamenov

Citation: Journal of Applied Physics 115, 17C735 (2014); doi: 10.1063/1.4868333

View online: http://dx.doi.org/10.1063/1.4868333

View Table of Contents: http://scitation.aip.org/content/aip/journal/jap/115/17?ver=pdfcov

Published by the AIP Publishing

\section{Articles you may be interested in}

Structural, electrical, and magnetic properties of SrRuO3 thin films

Appl. Phys. Lett. 104, 081608 (2014); 10.1063/1.4866775

Enhanced magnetoelectric effect in La0.67Sr0.33MnO3/PbZr0.52Ti0.48O3 multiferroic nanocomposite films with a SrRuO3 buffer layer

J. Appl. Phys. 113, 164106 (2013); 10.1063/1.4803057

Structure and properties of epitaxial perovskite $\mathrm{Pb}(\mathrm{Zr0} 0.52 \mathrm{Ti0}$.48)O3/La0.7Sr0.3MnO3 heterostructures

J. Appl. Phys. 111, 07D718 (2012); 10.1063/1.3677866

Effect of oxygen partial pressure on the structural and magnetic properties of $\mathrm{Ba}(\mathrm{Fe} 0.5 \mathrm{Mn} 0.5) \mathrm{O} 3-\delta$ epitaxial thin films

J. Appl. Phys. 105, 07D904 (2009); 10.1063/1.3062950

Ru-doped La 0.6 Sr 0.4 Mn O 3 thin films as a coercivity tunable electrode for magnetic tunnel junctions Appl. Phys. Lett. 86, 192505 (2005); 10.1063/1.1923199

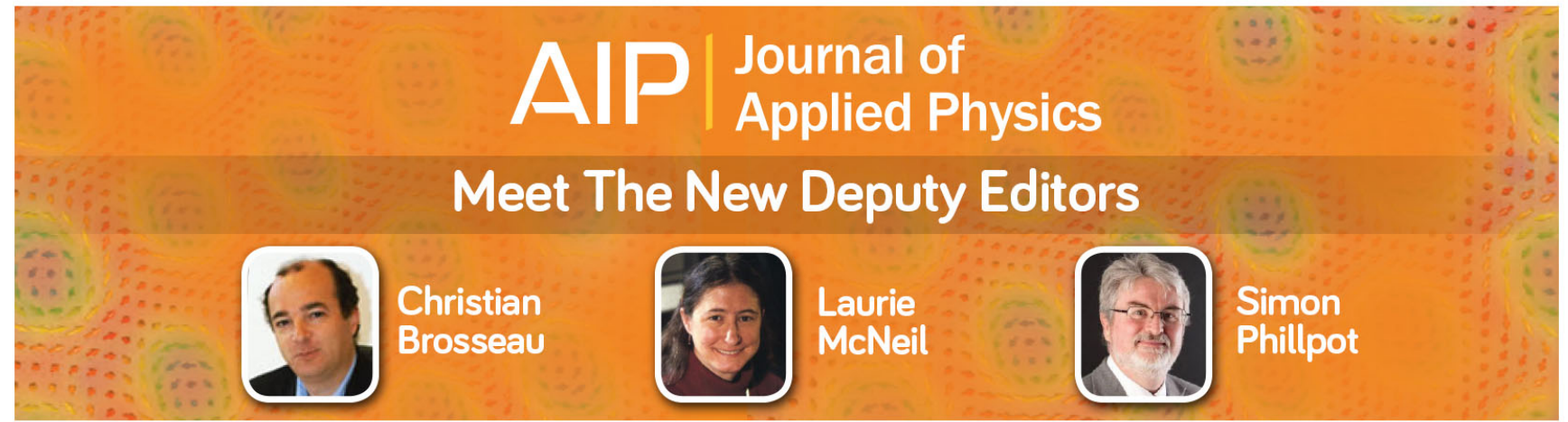




\title{
Magnetic, transport, and structural properties of $\mathrm{SrRuO}_{3}$ thin films
}

\author{
A. M. Majcher, ${ }^{1,2}$ K. Rode, ${ }^{1}$ J. M. D. Coey,${ }^{1}$ and P. Stamenov ${ }^{1, a)}$ \\ ${ }^{1}$ School of Physics and CRANN, Trinity College, Dublin 2, Ireland \\ ${ }^{2}$ Institute of Physics, Jagiellonian University Krakow, Krakow, Poland
}

(Presented 7 November 2013; received 24 September 2013; accepted 12 December 2013; published online 12 March 2014; corrected 13 March 2014)

\begin{abstract}
Thin films of the oxide ferromagnetic metal $\mathrm{SrRuO}_{3}(\mathrm{SRO})$ are studied, in view of their potential use within oxide-based spin electronic devices. Here, their epitaxial growth on Si-compatible $\mathrm{SrTiO}_{3}$ substrates is demonstrated by pulsed laser deposition. Detail on their initial, structural, magnetic, and magnetotransport characterisation is also provided. In particular, resistivity and Hall data are interpreted within a two/three carrier model, above $T_{\mathrm{C}}$. Below $T_{\mathrm{C}}$, some information about the anisotropy of the Fermi surface effective elipsoids $(\sim 1: 4)$ is recovered. The low-temperature saturation magnetisation approaches $1 \mu_{\mathrm{B}} / \mathrm{Ru}$, with an uniaxial anisotropy of above $0.6 \mathrm{MJm}^{-3}$. Despite its low $T_{\mathrm{C}}=160 \mathrm{~K}$, and in view of its relatively low carrier mobility, it is reasoned that SRO could be utilized as the electrode layer in low-temperature all-oxide-MTJ (Magnetic Tunnel Junction) demonstrators and as a seed layer for other ferromagnetic oxides, at and above room temperature. ㅇ 2014 AIP Publishing LLC. [http://dx.doi.org/10.1063/1.4868333]
\end{abstract}

Among other material combinations, the search is on, for thin film epitaxial sets that could enable the construction of all-oxide magnetic tunnel junctions for spin electronic applications. For a number of years, the ferromagnetic oxide metal $\mathrm{SrRuO}_{3}$ (SRO) has attracted attention ${ }^{1}$ as it can be grown in thin film form on $\mathrm{Si}$-compatible $\mathrm{SrTiO}_{3}$ (STO) substrates or seed layers, which could open the route towards such integration.

Here, we demonstrate the epitaxial growth of SRO thin films on non-intentionally miscut [001]-oriented STO substrates (heated to $\sim 700^{\circ} \mathrm{C}$ ) by pulsed laser deposition (PLD), and carry out structural, magnetic, and magnetotransport characterisation of the same. It should be noted that other methods have been also used successfully for the growth of this system, for example, RF reactive magnetron sputtering. ${ }^{1}$ SRO grows with the orthorhombic [110] direction normal to the substrate. By reciprocal space mapping, we have identified two distinct in-plane mosaics with the SRO [001] parallel to STO [100] and STO [010], respectively. There is a misalignment of $\sim 0.2^{\circ}$ between SRO [001] and STO [110], suggesting that strain is preserved for the entire thickness $(>100 \mathrm{~nm})$ of the films, perpendicular to the substrate. The films are close to atomically flat, with welldeveloped terraced morphology, with terrace walls primarily oriented along the (100) family of directions, with rather uniform step-height of $\sim 7 \mathrm{~nm}$.

The macroscopic magnetisation of the films is studied by inductive VSM and superconducting quantum interference device SQUID-based magnetometry below RT in fields of up to $\mu_{0} H \leq 14 \mathrm{~T}$. The Curie temperature $\left(T_{\mathrm{C}}\right)$ is determined by ZFC/FC (Zero-Field-Cooled/Field-Cooled) thermal scans in an applied field of $\mu_{0} H=10 \mathrm{mT}$, to be $T_{\mathrm{C}}$ $\simeq 150 \mathrm{~K}$. The low- $T\left(T \ll T_{\mathrm{C}}\right)$ saturation magnetisation of the films is $M_{\text {sat }}=24.6 \mathrm{Am}^{2} \mathrm{~kg}^{-1}\left(1.05 \mu_{\mathrm{B}} / \mathrm{f}\right.$.u. $)$, equivalent to a polarisation of $0.161 \mathrm{MA} / \mathrm{m}$. The approach to saturation

\footnotetext{
${ }^{\text {a) }}$ stamenov.plamen@tcd.ie.
}

$\left(4 \mathrm{~T} \leq \mu_{0} H \leq 14 \mathrm{~T}\right)$ is fitted to a non-linear model, allowing for a simple sinusoidal texture function, to yield an intrinsic uniaxial anisotropy constant of $K_{\mathrm{u}}=0.63 \mathrm{MJm}^{-3}$, while the apparent one is $K_{\text {eff }}=0.05 \mathrm{MJm}^{-3}$. The texture appears to be offset from the normal by about $33^{\circ}$, as a result of strain. Other studies have previously revealed very similar values ${ }^{2}$ $\left(35^{\circ}\right)$.

The high-field magnetisation and magneto-transport in SRO have been investigated before, ${ }^{3}$ however, no analysis is available of all accessible carrier types, besides some suggestions and indirect support for a two-band model of the electronic structure for SRO grown on $\mathrm{LaAl}_{3}{ }^{4}{ }^{4}$ Therefore, here, resistivity and Hall data are analysed within two/three-carrier models, in the temperature region above $T_{\mathrm{C}}(140-300 \mathrm{~K})$, with an applied field of up to $\mu_{0} H \leq 14 \mathrm{~T}$.

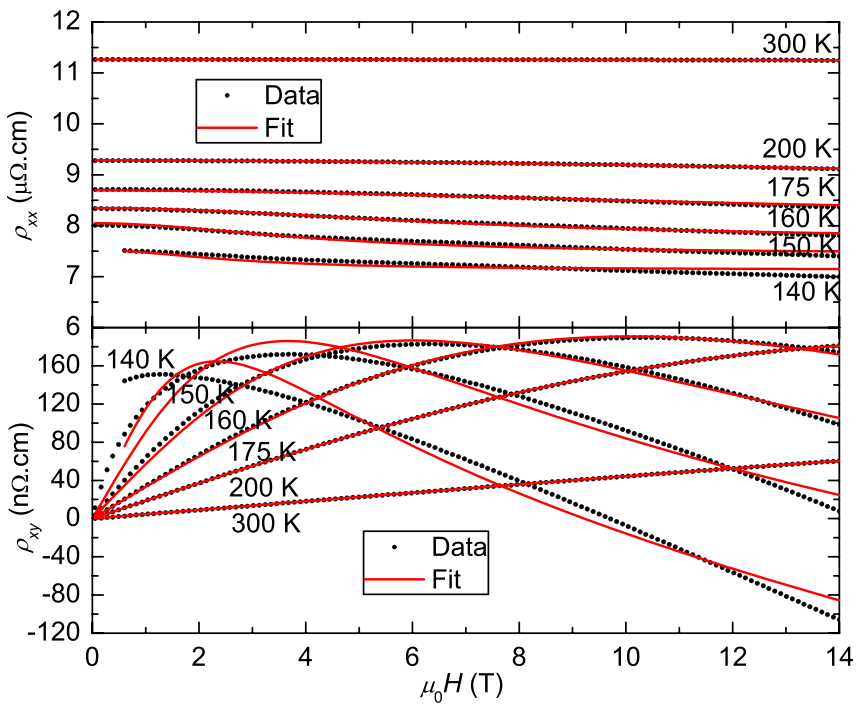

FIG. 1. Resistivity (top) and Hall resistivity (bottom), at selected temperatures around and above $T_{\mathrm{C}}$. The lines are fit to the model described in the text. 


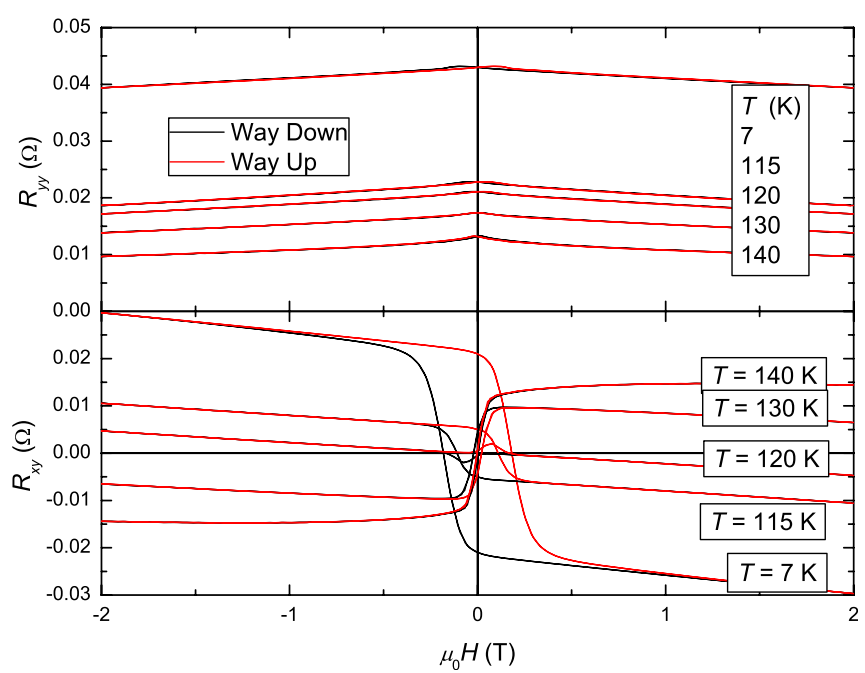

FIG. 2. Resistivity and Hall resistivity for an SRO thin film, at lowtemperature and in the vicinity of $T_{\mathrm{C}}$.

The interpretation suggested here is validated by highquality fits in a rather broad field and temperature interval, as illustrated in Fig. 1. The carrier concentrations vary by a factor of ten and are about $6 \times 10^{28}$ (majority $p$ ), $1 \times 10^{25}$ (majority $n$ ), and $7 \times 10^{25} \mathrm{~m}^{-3}$ (minority $p$ ) at $140 \mathrm{~K}$. The corresponding scattering times are $3 \times 10^{-14}, 5 \times 10^{-12}$, and $1 \times 10^{-12} \mathrm{~s}$, respectively.

Information somewhat complementary to the one accessible from bulk magnetometry can be extracted from analysing the detailed behaviour of the diagonal and the Hall resistance, as illustrated in Fig. 2. Fits to the highly nonlinear and dominated by spontaneous scattering curves are obviously challenging, however, it is still instructive to follow the temperature evolutions of the electronic and magnetic parameters as shown in Fig. 3. It is clearly seen that the change of sign of the spontaneous scattering coefficient occurs at a temperature significantly lower than $T_{\mathrm{C}}$.

The high-field magnetoresistance (1) exhibits no ShdH oscillations, however, it contains valuable information about the anisotropy of the Fermi surface and the scattering coefficients. The anisotropy of the magnetoresistance has been shown somewhat complicated, ${ }^{5}$ deviating substantially from

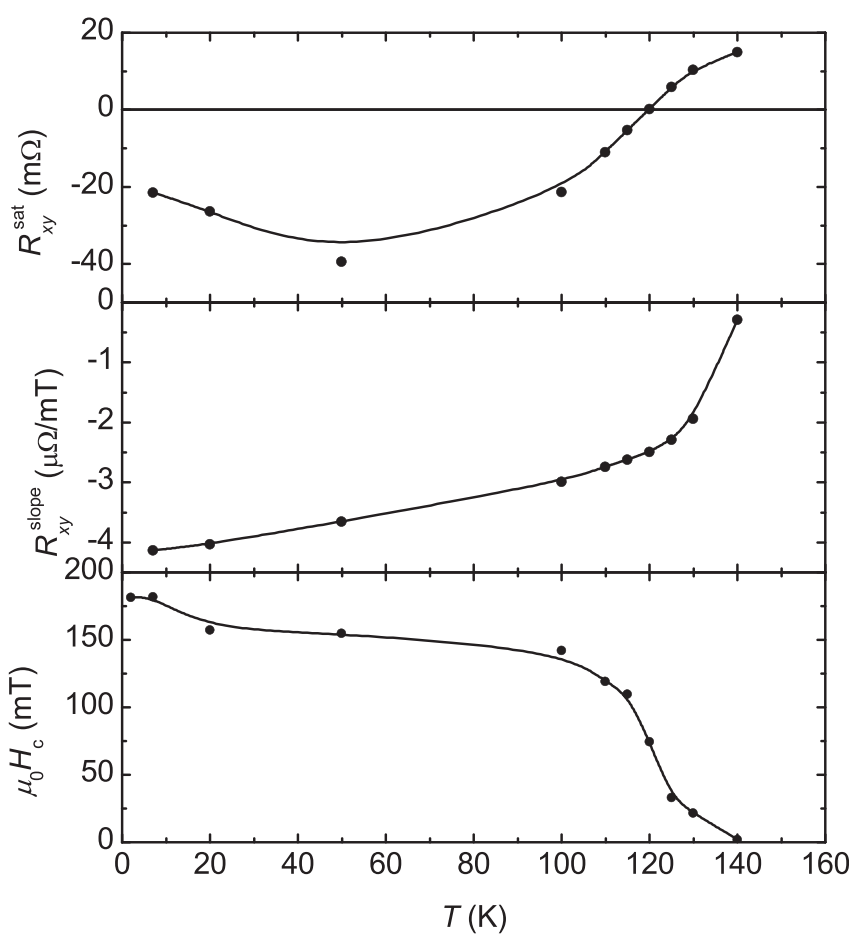

FIG. 3. Coercive field $\mu_{0} H_{\mathrm{c}}$, high-field Hall resistance slope $R_{x y}^{\text {slope }}$, and saturating Hall resistance $R_{x y}^{\text {sat }}$ for an SRO thin film. Note the different crossover temperatures.

the simple $\sin ^{2} \theta$ scaling, at low-temperatures. Other studies have interpreted the anisotropy as displaying distinctive characteristics of, and acting as a fingerprint for, monoclinic symmetry. ${ }^{2}$ The complicated field, angular, and temperature dependencies of the magnetoresistance and Hall coefficients have never been studied in sufficient detail and interpreted, for a single set of samples, in the entire temperature interval, both below and above $T_{\mathrm{C}}$. Here, an analysis is suggested, based on the observation of two distinct in-plane textures, and well-developed rhombohedral symmetry. The observed anisotropic profiles are shown, together with their modelling, in Fig. 4. The apparent anisotropy of $\sim 0.64(\sim 1 / 2)$, in combination with the observed crystallographic phase fraction of $0.465(1 / 1.15)$ allows for the deduction of the actual Fermi
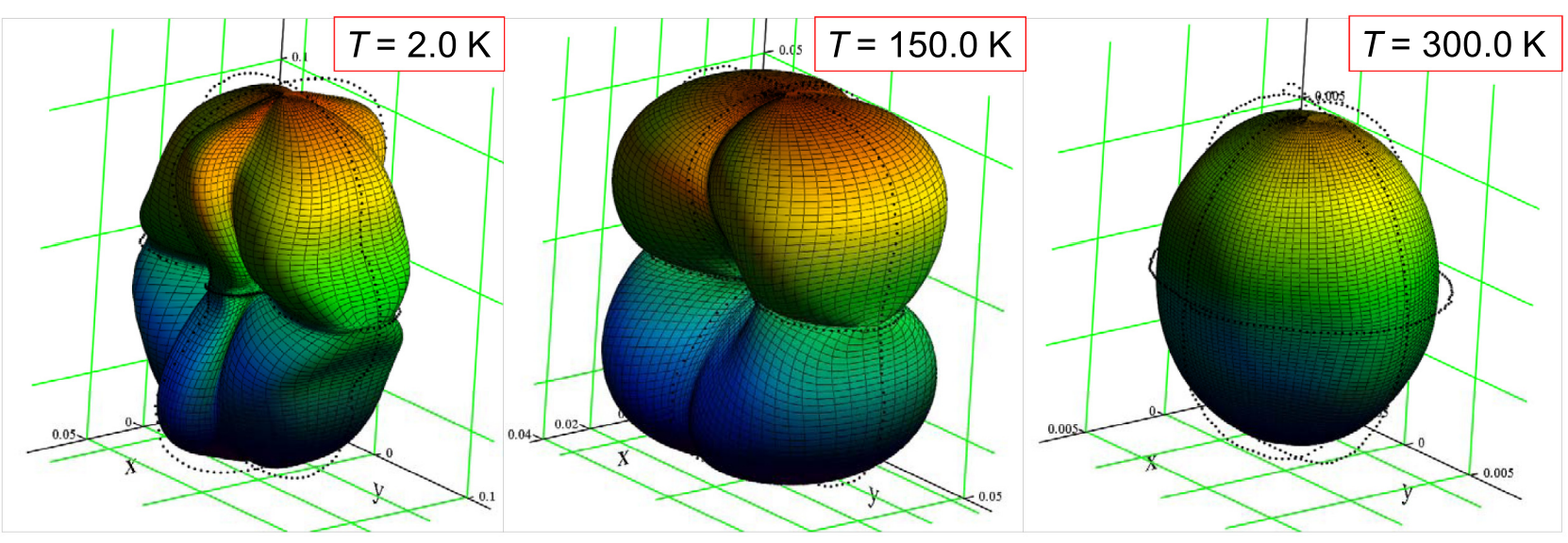

FIG. 4. Anisotropy of the high-field $\mu_{0} H=14 \mathrm{~T}$ magnetoresistance, at three different temperatures, well below, close to, and well above $T_{\mathrm{C}}$. The dots are experimental data, scaled by a factor of 1.1 , for visibility. 


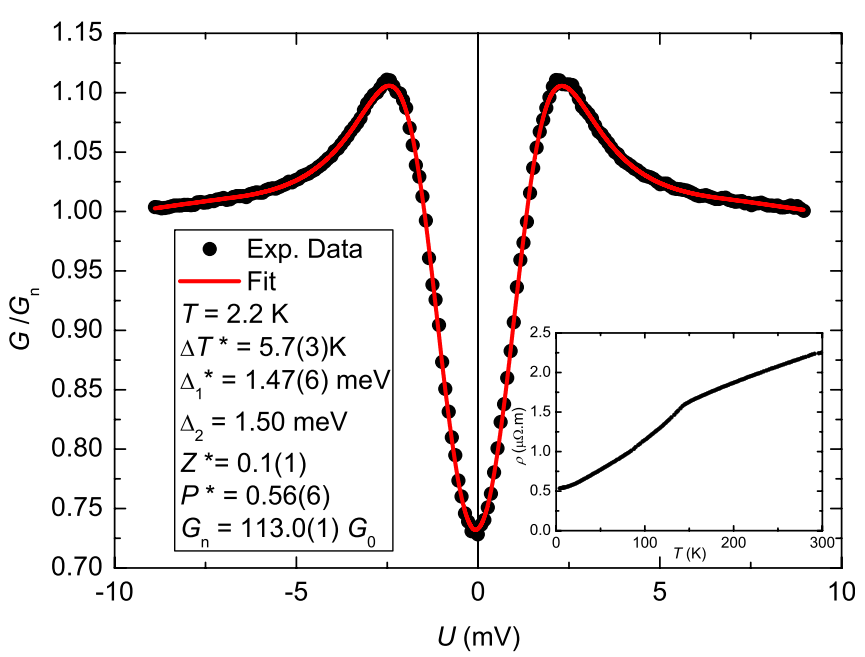

FIG. 5. Conductance spectra of a Nb/SRO contact at $\mu_{0} H=0.0 \mathrm{mT}$ and the modified BTK fit to it. The inset shows the temperature dependence of the resistivity for the same sample.

surface anisotropy ratio. The same is found to be as high as $0.82(\sim 1: 4)$.

The sample used for PCAR is prepared by conventionally shorting the majority of the available $5 \times 5 \mathrm{~mm}^{2}$ area with highly conductive paste, in order to minimize the series resistance contribution. A polyscrystalline niobium tip is used to form contacts at lattice temperatures in the range of 1.8 to $15 \mathrm{~K}$, in a $\mathrm{He}$ vapour-flow through sample space cryostat (Oxford instruments). Magnetic field, of up to $\mu_{0} H=1 \mathrm{~T}$, is applied in an arbitrary direction within the film plane, by a "Multimag" permanent-magnet-based variable-flux source. The construction of the PCAR setup and a description of the real-time derivative-spectrum measurement system used, and the spectral fitting software are can be found in references ${ }^{6-8}$ and are omitted here.

Fig. 5 presents an example of a typical derivative spectrum, together with its fitting and interpretation. It should be noted that all observed spectra are successfully fitted within the framework of the well-known modified BTK (Blonder, Tinkham and Klapwijk) ${ }^{9}$ theory, using the methodology attributed to, ${ }^{10}$ further taking into account superconducting proximity, electronic heating, modulation broadening, and some (rather small in this particular case) series resistance corrections. The Fermi level spin polarisation is inferred to be $|P|=0.56(6)$, and is constant in the temperature interval 1.9-9.0 K. Proximity gaps are typically only weakly pronounced $\Delta_{1} \sim 1.5 \mathrm{meV}$. Delta-barrier strengths observed are medium $Z \sim 0.1$, suggesting a diffusive model-based interpretation.

The maximal available magnetic field of up to $\mu_{0} H<1 \mathrm{~T}$ is clearly too weak to saturate the sample magnetically (even within the film plane) and is only altering the observed conductance spectra significantly in the vicinity of $T_{\mathrm{C}}=9.2 \mathrm{~K}$, by broadening the spectral features, much comparable to the thermal one. It should be noted that in virtually all observed spectra effective electronic temperatures $T_{\mathrm{e}}$ are well in excess of lattice ones, with a $\Delta T$ often in excess of $5 \mathrm{~K}$. This is attributed to an additional effective barrier formed at the top interface, exposed to ambient conditions, but does not preclude the correct interpretation of the spectra.

The characterisation of the electronic structure of SRO (in particular, the orthorhombic $\mathrm{SrRuO}_{3}$ ) is far from complete. The growth of significantly higher mobility single-crystalline films (the successful growth of bulk single crystals is less likely) combined with the use of very high magnetic fields $(>20 \mathrm{~T})$ could yield enough information for the experimental reconstruction of the Fermi surface for the majority holes. Access to the minority carriers is likely to require the use of chemical doping, in order to shift the Fermi level up sufficiently. In view of its relatively low carrier mobility, SRO could be utilized as the electrode layer in low-temperature all-oxide-MTJ demonstrators and as a seed layer for other ferromagnetic oxides, at and above room temperature.

The help of M. Venkatesan with low-field magnetometry is gratefully acknowledged, as is financial support from Science Foundation Ireland, within the SSPP (11/SIRG/I2130), NISE (10/IN1/I3002), and from the EU within the IFOX network.

${ }^{1}$ H. C. Yang, S. H. Liu, L. M. Wang, and H. E. Horng, J. Appl. Phys. 85, 5792 (1999).

${ }^{2}$ M. Ziese, I. Vrejoiu, and D. Hesse, Phys. Rev. B 81, 184418 (2010).

${ }^{3}$ M. Izumi, K. Nakazawa, Y. Bando, Y. Yoneda, and H. Terauchi, J. Phys. Soc. Jpn. 66, 3893 (1997).

${ }^{4}$ J. H. Cho, Q. X. Jia, X. D. Wu, S. R. Foltyn, and M. P. Maley, Phys. Rev. B 54, 37 (1996).

${ }^{5}$ O. Morán, W. Saldarriaga, and E. Baca, Solid State Sci. 11, 1187 (2009).

${ }^{6}$ P. Stamenov and J. M. D. Coey, J. Appl. Phys. 109, 07 C713 (2011).

${ }^{7}$ P. Stamenov, J. Appl. Phys. 111, 07C519 (2012).

${ }^{8}$ P. Stamenov, J. Appl. Phys. 113, 17C718 (2013).

${ }^{9}$ G. E. Blonder, M. Tinkham, and T. M. Klapwijk, Phys. Rev. B 25, 4515 (1982).

${ }^{10}$ R. J. Soulen, J. M. Byers, M. S. Osofsky, B. Nadgorny, T. Ambrose, S. F. Cheng, P. R. Broussard, C. T. Tanaka, J. Nowak, J. S. Moodera, A. Barry, and J. M. D. Coey, Science 282, 85 (1998). 